\title{
On classes of meromorphic functions with fixed point and fixed second and finitely many coeeficients defined by $q$-Derivative
}

\author{
G. E. Abo Elyazyd ${ }^{1}$ A. M. Shahin ${ }^{2}$ and H. E. Darwish ${ }^{3}$ \\ 1,2,3 Department of Mathematics Faculty of Science, Mansoura University Mansoura, 35516, Egypt.
}

Received 11-04-2021, Accepted 10-05-2021, Published 20-06-2021.

DOI: 10.52113/2/08.01.2021/121-129

\begin{abstract}
In this paper we consider the class $M_{q} S^{*}(\zeta, \alpha, c) \quad$ which consisting of meromorphic univalent functions with a fixed point $\geqslant$ in $U^{*}:=\{z: z \in \mathrm{C}, 0<|z|<1\}$ and with fixed second positive coefficient. The aim of the present paper is to drive several interesting properties as coefficient estimates, distortion theorems, radii of starlikeness and convexity and closure theorems of $f(z)$ in the class $M_{q} S^{*}(\zeta, \alpha, c)$. The results are generalized to families with finitely many fixed coefficients.
\end{abstract}

2010 Mathematics Subject Classification: $30 \mathrm{C} 45$.

Key words: Meromorphic functions, fixed second positive coefficient, starlike function, convex function, closure theorem, Quantam calculus.

\section{Introduction}

The applications of $q$-calculus are new area in the last 25 years. This great interest is due to its application in various branches of mathematics and physics. The first applications of $q$-calculus was made by Jackson [9, 10]. He developed $q$ derivative and $q$-integral in a systematic. Aral and Gupta [5, 1, 7] defined the $q$ analogue of Baskakov Durrmeyer operator which based on $q$-analogue of beta function. The authors studied approximation and geometric properties of these $q$ operators in some subclasses of analytic functions in compact disk. Studies on quantum groups have played an important role in defining geometrical interpretation of $q$-analysis. It also suggests a relation between integrable systems and $q$-analysis.
Another important $q$-generalization of complex operators is $q$-Picard and $q$ Gauss-Weierstrass singular integral operators discussed in $[1,2,3]$. Other $q$ analogues of differential operators have been introduced in [11].

Let $>$ be a fixed point in the punctured unit disk $U^{*}=\{z \in \mathrm{C}, 0<|z|<1\}=U /\{0\}$.

Denote by $M(\zeta)$ be the class of meromorphic functions of the form

$$
f(z)=\frac{1}{z-\zeta}+\sum_{k=1}^{\infty} a_{k}(z-\zeta)^{k}, \quad z \neq \zeta .
$$

A function $f(z)$ of the form (1.1) is in the class of meromorphic starlike of order $\alpha$ $(0 \leq \alpha<1)$ denoted by $S(\zeta, \alpha)$, if

$$
R\left\{-\frac{(z-\zeta) f^{\prime}(z)}{f(z)}\right\}>\alpha,(z-\zeta) \in U
$$


and is in the class of meromorphic convex of order $\alpha \quad(0 \leq \alpha<1)$ denoted by $K(\zeta, \alpha)$, if

$$
R\left\{-\left(1+\frac{(z-\zeta) f^{\prime \prime}(z)}{f^{\prime}(z)}\right)\right\}>\alpha, \quad(z-\zeta) \in U
$$

We recall some concepts of $q$-calculus, all details about $q$-calculus used in this paper can be found in $[4,8]$, for $n \in \mathrm{N}$, the q-number is defined as follows:

$$
[k]_{q}=\frac{1-q^{k}}{1-q}, \quad 0<q<1 .
$$

Hence, $[k] q$ can be expressed as a geometric series $\sum_{i=0}^{k-1} q^{i}$, when $k \rightarrow \infty$ the series converges to $\frac{1}{12}$. As $q$ (b) 1 , $[k] \rightarrow k$. The $q$-derivative of a function $\mathrm{f}(\mathrm{z})$ is defined by

$$
D_{q} f(z)=\frac{f(z)-f(q z)}{(1-q) z}, \quad(q \neq 1, z \neq 0)
$$

and $D_{q} f(0)=f^{\prime}(0)$ and $D_{q}^{2} f(z)=D_{q}\left(D_{q} f(z)\right)$. For a function $g(z)=z^{k}$ we found that

$$
D_{q}(g(z))=D_{q} z^{k}=\frac{\left(1-q^{k}\right)}{(1-q)} z^{k-1}=[k]_{q} z^{k-1}
$$

and

$$
\lim _{q \rightarrow 1^{-}} D_{q}(g(z))=\lim _{q \rightarrow 1^{-}}[k]_{q} z^{k-1}=k z^{k-1}=g^{\prime}(z),
$$

where $g$ is the ordinary derivative. The q-Jackson definite integral of the function $f(z)$ is defined by

$$
\int_{0}^{z} f(t) d_{q} t=(1-q) z \sum_{n=0}^{\infty} f\left(z q^{n}\right) q^{n}, \quad z \in \mathrm{C} .
$$

Definition 1: A function $f(z)$ given by (1.1) is in the class $M_{q} S(\zeta, \alpha)$ of meromorphically starlike functions of order $\epsilon_{\text {in }} U^{\oplus}$ if it satisfies

$$
\operatorname{Re}\left\{-\frac{q(z-\zeta) D_{q} f(z)}{f(z)}\right\}>\alpha \quad((z-\zeta) \in U, 0<q<1,0 \leq \alpha
$$

Let $M_{p}(\zeta)$ denote the subclass of $M(\zeta)$ consisting of functions of the form

$$
f(z)=\frac{1}{z-\zeta}+\sum_{k=1}^{\infty} a_{k}(z-\zeta)^{k}, \quad\left(a_{k} \geq 0\right) .
$$

Also, we define the class $M_{q} S^{*}(\zeta, \alpha)$ by

$$
M_{q} S^{*}(\zeta, \alpha)=M_{q} S(\zeta, \alpha) \cap M_{p}(\zeta) .
$$

\section{Coefficient Estimates}

Theorem 1: Let the function $f \in M_{p}(\zeta)$ be given by (1.4). Then $f \in M_{q} S^{*}(\zeta, \alpha)$ if and only if

$$
\sum_{k=1}^{\infty}\left(q[k]_{q}+\alpha\right) a_{k} \leq 1-\alpha \quad\left(a_{k} \geq 0\right),
$$

where $0<q<1$ and $0 \leq \alpha<1$. The result is sharp.

Proof: Let $f(z) \in M_{q} S^{*}(\zeta, \alpha)$. Then in view of (1.3), we get

$$
\operatorname{Re}\left\{-\frac{q(z-\zeta) D_{q} f(z)-\alpha f(z)}{f(z)}\right\} \geq 0 .
$$

A simple computation yields

$$
\operatorname{Re}\left\{\frac{\frac{1-\alpha}{z-\zeta}-\sum_{k=1}^{\infty}\left(q[k]_{q}+\alpha\right) a_{k}(z-\zeta)^{k}}{\frac{1}{z-\zeta}+\sum_{k=1}^{\infty} a_{k}(z-\zeta)^{k}}\right\} \geq 0 .
$$

The above condition must hold for all values of $(z-\zeta)$ in $U$ if we choose $(z-\zeta)$ to be the real and $(z-\zeta) \rightarrow 1^{-}$we get

$$
\begin{aligned}
& \frac{(1-\alpha)-\sum_{k=1}^{\infty}\left(q[k]_{q}+\alpha\right) a_{k}}{1+\sum_{k=1}^{\infty} a_{k}} \geq 0 \\
& (1-\alpha)-\sum_{k=1}^{\infty}\left(q[k]_{q}+\alpha\right) a_{k} \geq 0
\end{aligned}
$$




$$
\sum_{k=1}^{\infty}\left(q[k]_{q}+\alpha\right) a_{k} \leq(1-\alpha) .
$$

Conversely, by applying the hypothesis (2.1) and choosing $|z-\zeta|=r<1$ we find that

$$
\operatorname{Re}\left\{-\frac{q(z-\zeta) D_{q} f(z)}{f(z)}\right\} \geq \frac{\alpha\left(1+\sum_{k=1}^{\infty} a_{k}\right)}{1+\sum_{k=1}^{\infty} a_{k}}=\alpha .
$$

Then $f \in M_{q} S^{*}(\zeta, \alpha)$.

Lemma 1: Let function $f(z)$ given by (1.4) be in the class $M_{q} S^{*}(\zeta, \alpha)$, which satisfies

$$
a_{k} \leq \frac{1-\alpha}{q[k]_{q}+\alpha}
$$

Hence we can take

$$
a_{1} \leq \frac{1-\alpha}{q+\alpha}
$$

Let $M_{q} S^{*}(\zeta, \alpha, c)$ denote the class of functions $f(z)$ in $M_{q} S^{*}(\zeta, \alpha)$ of the form

$$
f(z)=\frac{1}{z-\zeta}+\frac{(1-\alpha) c}{(q+\alpha)}(z-\zeta)+\sum_{k=2}^{\infty} a_{k}(z-\zeta)^{k}
$$

where $0<q<1,0 \leq \alpha<1$ and $0<c<1$.

Theorem 2: Let the function $f(z)$ be defined by (2.2). Then $f(z) \in$ $M_{q} S^{*}(\zeta, \alpha, c)$ if and only if

$$
\sum_{k=2}^{\infty}\left(q[k]_{q}+\alpha\right) a_{k} \leq(1-\alpha)(1-c) .
$$

The result is sharp.

Proof: Putting

$$
a_{1} \leq \frac{c(1-\alpha)}{q+\alpha}, \quad 0 \leq c \leq 1
$$

in (2.1), and simplifying we get the result.
The result is sharp for the function

$$
f(z)=\frac{1}{z-\zeta}+\frac{(1-\alpha) c}{(q+\alpha)}(z-\zeta)+\frac{(1-c)(1-\alpha)}{\left(q[k]_{q}+\alpha\right)}(z-\zeta)^{k},
$$

Corollary: Let the function $f(z)$

defined by (2.2) be in the class

$M_{q} S^{*}(\zeta, \alpha, c)$. Then

$$
a_{k} \leq \frac{(1-c)(1-\alpha)}{\left(q[k]_{q}+\alpha\right)} \quad(k \geq 2) \text {. }
$$

The result is sharp for the function $f(z)$ given by (2.5).

\section{Closure Theorems}

Theorem 3: The class $M_{q} S^{*}(\zeta, \alpha, c)$ is closed under convex linear combination.

Proof: Let the function $f(z)$ be defined by (2.2). Define the function $h(z)$ by

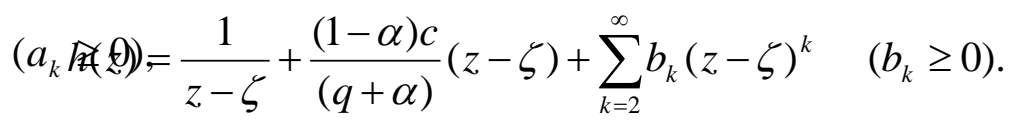

Suppose that $f(z)$ and $h(z)$ are in the class $M_{q} S^{*}(\zeta, \alpha, c)$, it is enough to prove that the function

$$
H(z)=\lambda f(z)+(1-\lambda) h(z) \quad(0 \leq \lambda \leq 1)
$$

also be in the class $M_{q} S^{*}(\zeta, \alpha, c)$.

Since

$$
H(z)=\frac{1}{z-\zeta}+\frac{(1-\alpha) c}{(q+\alpha)}(z-\zeta)+\sum_{k=2}^{\infty}\left\{\lambda a_{k}+(1-\lambda) b_{k}\right\}(z-\zeta)^{k}
$$

we obtain that

$\sum_{k=2}^{\infty}\left(q[k]_{q}+\alpha\right)\left\{\lambda a_{k}+(1-\lambda) b_{k}\right\} \leq(1-\alpha)(1-c)$

by returning to the Theorem 2 . Hence $H(z) \in M_{q} S^{*}(\zeta, \alpha, c)$. This clearly 
completes the proof of the Theorem.

Theorem 4: Let the functions $f_{j}(z)=\frac{1}{z-\zeta}+\frac{(1-\alpha) c}{(q+\alpha)}(z-\zeta)+\sum_{k=2}^{\infty} a_{k, j}(z-\zeta)^{k}$,

be in the class $M_{q} S^{*}(\zeta, \alpha, c)$ for every $j=1,2, \ldots, m$. Then the function $F(z)$ defined by

$$
F(z)=\sum_{j=1}^{m} d_{j} f_{j}(z) \quad\left(d_{j} \geq 0\right)
$$

is also in the class $M_{q} S^{*}(\zeta, \alpha, c)$, where

$$
\sum_{j=1}^{m} d_{j}=1 \text {. }
$$

Proof: From (3.5), (3.6) and (3.7), we $F(z)=\sum_{j=1}^{m} d_{j} f_{j}(z)=\frac{1}{z-\zeta}+\frac{(1-\alpha) c}{(q+\alpha)}(z-\zeta)+\sum_{k=2}^{\infty}\left(\sum_{j=1}^{m} d_{j} a_{k, j}\right) \frac{1}{z-\zeta}+\frac{(1-\alpha) c}{(z-\zeta)^{k}}(z-\zeta)+\sum_{k=2}^{\infty} \frac{\lambda_{k}(1-c)(1-\alpha)}{\left(q[k]_{q}+\alpha\right)}(z-\zeta)^{k}$,

Since $f_{j}(z) \in M_{q} S^{*}(\zeta, \alpha, c)$ for every $\mathrm{j}=1,2, \ldots, \mathrm{m}$, in view of Theorem 2 , we get $\sum_{k=2}^{\infty}\left(q[k]_{q}+\alpha\right) a_{k, j} \leq(1-\alpha)(1-c), \quad j=1,2, \ldots, m$.

Thus we obtain since

Proof : We suppose that the function $f(z)$ can be expressed in the form (3.12). Then from (3.10), (3.11) and (3.13), we have

$$
\begin{aligned}
& \sum_{k=2}^{\infty} \frac{\lambda_{k}(1-c)(1-\alpha)}{\left(q[k]_{q}+\alpha\right)} \cdot\left(q[k]_{q}+\alpha\right) \\
= & (1-c)(1-\alpha) \sum_{k=2}^{\infty} \lambda_{k} \\
= & (1-c)(1-\alpha)(1-\lambda) \\
\leq & (1-c)(1-\alpha) .
\end{aligned}
$$

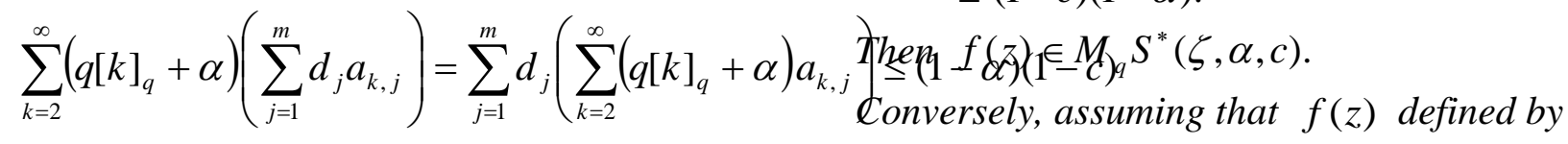

which implies that $F(z) \in M_{q} S^{*}(\zeta, \alpha, c)$.

Theorem 5: Let

$$
f_{1}(z)=\frac{1}{z-\zeta}+\frac{(1-\alpha) c}{(q+\alpha)}(z-\zeta)
$$

and
(2.2) be in the class $M_{q} S^{*}(\zeta, \alpha, c)$ which satisfies (2.6), we obtain

\section{Setting}

$$
a_{k} \leq \frac{(1-c)(1-\alpha)}{\left(q[k]_{q}+\alpha\right)} \quad(k \geq 2) .
$$

$$
\lambda_{k}=\frac{\left(q[k]_{q}+\alpha\right)}{(1-c)(1-\alpha)} a_{k} \quad(k \geq 2),
$$

and 


$$
\lambda=1-\sum_{k=2}^{\infty} \lambda_{k}
$$

this gives (3.12). This compelets the proof of the Theorem.

Corollary 2: The extreme points of the class $M_{q} S^{*}(\zeta, \alpha, c)$ are the functions $f_{k}(z)(k \geq 1)$ given by (3.10) and (3.11) in Theorem 5 .

\section{Growth and Distortion Theorems}

Theorem 6: If the function $f(z)$

defined by (2.2) is in the class

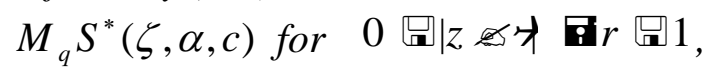
then we have

$$
\begin{aligned}
\frac{1}{r} & -\frac{(1-\alpha) c}{(q+\alpha)} r-\frac{(1-\alpha)(1-c)}{\left(q^{2}+q+\alpha\right)} r^{2} \leq|f(z)| \\
& \leq \frac{1}{r}+\frac{(1-\alpha) c}{(q+\alpha)} r+\frac{(1-\alpha)(1-c)}{\left(q^{2}+q+\alpha\right)} r^{2}
\end{aligned}
$$

with equality for

$$
f_{2}(z)=\frac{1}{z-\zeta}+\frac{(1-\alpha) c}{(q+\alpha)}(z-\zeta)+\frac{(1-\alpha)(1-c)}{\left(q^{2}+q+\alpha\right)}(z-\zeta)^{2} . \quad k a_{k} \leq \frac{k(1-\alpha)(1-c)}{\left(q[k]_{q}+\alpha\right)}, \quad k \geq 2 .
$$

with equality for

$$
f_{2}(z)=\frac{1}{z-\zeta}+\frac{(1-\alpha) c}{(q+\alpha)}(z-\zeta)+\frac{(1-\alpha)(1-c)}{\left(q^{2}+q+\alpha\right)}(z-\zeta)^{2} .
$$

Proof: Suppose that $f(z)$ is in the class $M_{q} S^{*}(\zeta, \alpha, c)$. By Theorem 2, we have Thus, for $0<|z-\zeta|=r<1$, we obtain

$$
a_{k} \leq \frac{(1-\alpha)(1-c)}{\left(q[k]_{q}+\alpha\right)}, \quad k \geq 2 \text {. }
$$

Thus, for $0<|z-\zeta|=r<1$

$$
\left|f^{\prime}(z)\right| \leq\left|\frac{-1}{(z-\zeta)^{2}}\right|+\frac{(1-\alpha) c}{(q+\alpha)}+\sum_{k=2}^{\infty} k a_{k}|z-\zeta|^{k-1}, \quad(|z-\zeta|=r)
$$$$
|f(z)| \leq \frac{1}{|z-\zeta|}+\frac{(1-\alpha) c}{(q+\alpha)}|z-\zeta|+\sum_{k=2}^{\infty} a_{k}|z-\zeta|^{k}, \quad \mid z-\zeta \leq=\frac{1 r}{r^{2}}+\frac{(1-\alpha) c}{(q+\alpha)}+\frac{(1-\alpha)(1-c)}{\left(q^{2}+q+\alpha\right)} r .
$$$$
\leq \frac{1}{r^{2}}+\frac{(1-\alpha) c}{(q+\alpha)}+r \sum_{k=2}^{\infty} k a_{k}
$$

$$
\begin{aligned}
& \leq \frac{1}{r}+\frac{(1-\alpha) c}{(q+\alpha)} r+r^{2} \sum_{k=2}^{\infty} a_{k} \\
& \leq \frac{1}{r}+\frac{(1-\alpha) c}{(q+\alpha)} r+\frac{(1-\alpha)(1-c)}{\left(q^{2}+q+\alpha\right)} r^{2}
\end{aligned}
$$

and

$$
\left|f^{\prime}(z)\right| \geq\left|\frac{-1}{(z-\zeta)^{2}}\right|-\frac{(1-\alpha) c}{(q+\alpha)}-\sum_{k=2}^{\infty} k a_{k}|z-\zeta|^{k-1}, \quad(|z-\zeta|=r)
$$

and

$$
|f(z)| \geq \frac{1}{|z-\zeta|}-\frac{(1-\alpha) c}{(q+\alpha)}|z-\zeta|-\sum_{k=2}^{\infty} a_{k}|z-\zeta|^{k}, \quad(|z-\zeta|=r)
$$




$$
\begin{aligned}
& \geq \frac{1}{r^{2}}-\frac{(1-\alpha) c}{(q+\alpha)}-r \sum_{k=2}^{\infty} k a_{k} \\
& \geq \frac{1}{r^{2}}-\frac{(1-\alpha) c}{(q+\alpha)}-\frac{(1-\alpha)(1-c)}{\left(q^{2}+q+\alpha\right)} r .
\end{aligned}
$$

The proof of the Theorem is complete.

\section{Radii of Starlikeness and Convexity}

Theorem 8: Let the function $f(z)$

defined by (2.2) is in the class

$M_{q} S^{*}(\zeta, \alpha, c)$. Then $f(z)$ is starlike of

order $\rho(0 \leq \rho \leq 1)$ in the disk

$|z-\zeta|<r_{1}(\zeta, \alpha, c, \rho)$, where $r_{1}(\zeta, \alpha, c, \rho)$

is the largest value for which

$\frac{(1+\rho)(1-\alpha) c}{(q+\alpha)} r^{2}+\frac{(1-\alpha)(1-c)(k+\rho)}{\left(q[k]_{q}+\alpha\right)} r^{k+1} \leq(1-\rho)$

for $k \geq 2$. The result is sharp for function $f(z)$ given by (2.5).

Proof : It suffices to show that

$$
\left|\frac{(z-\zeta) f^{\prime}(z)}{f(z)}+1\right| \leq(1-\rho)
$$

for $|z-\zeta|<r_{1}(\zeta, \alpha, c, \rho)$. We have

and it follows that from (2.3), we may take

$$
a_{k} \leq \frac{(1-c)(1-\alpha)}{\left(q[k]_{q}+\alpha\right)} \lambda_{k}, \quad k \geq 2
$$

where $\lambda_{k} \geq 0$ and $\sum_{k=2}^{\infty} \lambda_{k} \leq 1$.

For each fixed $r$, we choose the positive integer $k_{0}=k_{0}(r)$ for which $\frac{(k+\rho)}{\left(q[k]_{q}+\alpha\right)} r^{k+1}$ is maximal. Then it follows that

$$
\sum_{k=2}^{\infty}(k+\rho) a_{k} r^{k+1} \leq \frac{(1-c)(1-\alpha)\left(k_{0}+\rho\right)}{\left(q\left[k_{0}\right]_{q}+\alpha\right)} r^{k_{o}+1} \text {. }
$$

Then $f(z)$ is starlike of order $\rho$ in $0<|z-\zeta|<r_{1}(\zeta, \alpha, c, \rho)$ provided that

$\frac{(1+\rho)(1-\alpha) c}{(q+\alpha)} r^{2}+\frac{(1-\alpha)(1-c)\left(k_{0}+\rho\right)}{\left(q\left[k_{0}\right]_{q}+\alpha\right)} r^{k_{0}+1} \leq(1-\rho)$

we find the value $r_{0}=r_{0}(\zeta, \alpha, c, \rho)$ and the corresponding integer $k_{0}\left(r_{0}\right)$ so that

$$
\frac{(1+\rho)(1-\alpha) c}{(q+\alpha)} r_{0}^{2}+\frac{(1-\alpha)(1-c)\left(k_{0}+\rho\right)}{\left(q\left[k_{0}\right]_{q}+\alpha\right)} r^{k_{0}+1}=(1-\rho) \text {. }
$$

Then this value is the radius of starlikeness of order $\zeta$ for function $f$ belongs to class $\left|\frac{(z-\zeta) f^{\prime}(z)}{f(z)}+1\right|=\mid \frac{\frac{2(1-\alpha) c}{(q+\alpha)}(z-\zeta)+\sum_{k=2}^{\infty}(k+1) a_{k}(z-\zeta)^{k^{M}}}{\frac{1}{z-\zeta}+\frac{(1-\alpha) c}{q+\alpha}(z-\zeta)+\sum_{k=2}^{\infty} a_{k}(z-\zeta)^{k}} d$

Hence (5.2) holds true if $\frac{2(1-\alpha) c}{(q+\alpha)} r^{2}+\sum_{k=2}^{\infty}(k+1) a_{k} r^{k+1}$ $M_{q} S^{*}(\zeta, \alpha, c)$. Then $f(z)$ is convex of order $\rho(0 \leq \rho \leq 1)$ in the disk $|z-\zeta|<r_{2}(\zeta, \alpha, c, \rho)$, where $r_{2}(\zeta, \alpha, c, \rho)$ is the largest value for which

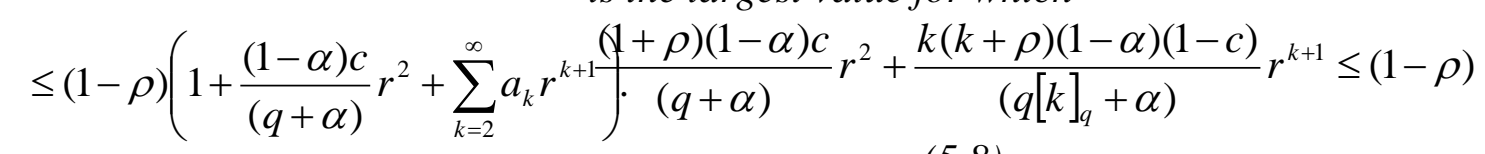

or

$$
\frac{(1+\rho)(1-\alpha) c}{(q+\alpha)} r^{2}+\sum_{k=2}^{\infty}(k+\rho) a_{k} r^{k+1} \leq(1-\rho)
$$

for $k \geq 2$. The result is sharp for function $f(z)$ given by (2.5).

Proof : It suffices to show that 


$$
\left|\frac{(z-\zeta) f^{\prime \prime}(z)}{f^{\prime}(z)}+2\right| \leq(1-\rho)
$$

for $|z-\zeta|<r_{2}$ with the aid of Theorem 2 .

Then by using the same technique in the proof of Theorem 8 we can complete the proof.

\section{The Class $M_{q} S^{*}\left(\zeta, \alpha, c_{n}\right)$}

In addition to fixing the second coefficient, we can fix finitely many coefficients.

Let $M_{q} S^{*}\left(\zeta, \alpha, c_{n}\right)$ denote the class of functions in $M_{q} S^{*}(\zeta, \alpha)$ of the form $f(z)=\frac{1}{z-\zeta}+\sum_{i=1}^{n} \frac{c_{i}(1-\alpha)}{\left(q[i]_{q}+\alpha\right)}(z-\zeta)^{i}+\sum_{k=n+1}^{\infty} a_{k}(z-\zeta)$

where $0 \leq \sum_{i=1}^{n} c_{i}=c \leq 1$. Note that $M_{q} S^{*}\left(\zeta, \alpha, c_{1}\right)=M_{q} S^{*}(\zeta, \alpha, c)$.

\section{7. properties of}

$$
M_{q} S^{*}\left(\zeta, \alpha, c_{n}\right)
$$

Theorem 10: (coefficient estimates) A function $f \mathbf{Q}$ defined by (6.1) is in $M_{q} S^{*}\left(\zeta, \alpha, c_{n}\right)$ if and only if

$$
\sum_{k=n+1}^{\infty}\left(q[k]_{q}+\alpha\right) a_{k} \leq(1-\alpha)\left(1-\sum_{i=1}^{n} c_{i}\right),
$$

where $0 \leq \sum_{i=1}^{n} c_{i}=c \leq 1$ and $0 \diamond c_{i} \diamond 1$.

The result is sharp.

Proof : Putting

$$
a_{i} \leq \frac{c_{i}(1-\alpha)}{\left(q[i]_{q}+\alpha\right)}, \quad i=1,2,3, \ldots, n,
$$

in (2.1), and simplifying we get the result.

The result is sharp for the function

$$
f(z)=\frac{1}{z-\zeta}+\sum_{i=1}^{n} \frac{(1-\alpha) c_{i}}{\left(q[i]_{q}+\alpha\right)}(z-\zeta)^{i}+\frac{\left(1-\sum_{i=1}^{n} c_{i}\right)(1-\alpha \text { Theorem 12: Let the functions }}{\left(q[k]_{q}+\alpha\right) f_{j}}(z)=\frac{\zeta)^{k} 1}{z-\zeta}+\sum_{i=1}^{k} \frac{n \boldsymbol{t}_{i} 1(1-\alpha)}{\left(q[i]_{q}+\alpha\right)}(z-\zeta)^{i}+\sum_{k=n+1}^{\infty} a_{k, j}(z-\zeta)^{k},
$$

Corollary 3: Let the function $f(z)$ defined by (6.1) be in the class $M_{q} S^{*}\left(\zeta, \alpha, c_{n}\right)$. Then

$$
a_{k} \leq \frac{\left(1-\sum_{i=1}^{n} c_{i}\right)(1-\alpha)}{\left(q[k]_{q}+\alpha\right)}, \quad(k \geq n+1) .
$$

The result is sharp for the function $f(z)$ given by (7.1).

Theorem 11: The class $M_{q} S^{*}\left(\zeta, \alpha, c_{n}\right)$ is closed under convex linear combination.
'Proof : Let the function $f(z)$ be defined by (6.1). Define the function $h(z)$ by

$$
h(z)=\frac{1}{z-\zeta}+\sum_{i=1}^{n} \frac{c_{i}(1-\alpha)}{\left(q[i]_{q}+\alpha\right)}(z-\zeta)^{i}+\sum_{k=n+1}^{\infty} b_{k}(z-\zeta)^{k} .
$$

Suppose that $f(z)$ and $h(z)$ are in the class $M_{q} S^{*}\left(\zeta, \alpha, c_{n}\right)$, it is enough to prove that the function

$$
H(z)=\lambda f(z)+(1-\lambda) h(z) \quad(0 \leq \lambda \leq 1)
$$

also be in the class $M_{q} S^{*}\left(\zeta, \alpha, c_{n}\right)$.

Since

$$
H(z)=\frac{1}{z-\zeta}+\sum_{i=1}^{n} \frac{c_{i}(1-\alpha)}{\left(q[i]_{q}+\alpha\right)}(z-\zeta)^{i}+\sum_{k=n+1}^{\infty}\left\{\lambda a_{k}+(1-\lambda) b_{k}\right\}
$$

we obtain that

$$
\sum_{k=n+1}^{\infty}\left(q[k]_{q}+\alpha\right)\left\{\lambda a_{k}+(1-\lambda) b_{k}\right\} \leq(1-\alpha)\left(1-\sum_{i=1}^{n} c_{i}\right)
$$

by returning to the Theorem 10 . Hence $H(z) \in M_{q} S^{*}\left(\zeta, \alpha, c_{n}\right)$. This clearly completes the proof of the Theorem. 
be in the class $M_{q} S^{*}\left(\zeta, \alpha, c_{n}\right)$ for every $j=1,2, \ldots, m$. Then the function $F(z)$ defined by

$$
F(z)=\sum_{j=1}^{m} d_{j} f_{j}(z) \quad\left(d_{j} \geq 0\right)
$$

is also in the class $M_{q} S^{*}\left(\zeta, \alpha, c_{n}\right)$, where

$$
\sum_{j=1}^{m} d_{j}=1 \text {. }
$$

Proof : From (7.3), (7.4) and (7.5) we have

$$
\begin{aligned}
& F(z)=\sum_{j=1}^{m} d_{j} f_{j}(z)=\frac{1}{z-\zeta}+\sum_{i=1}^{n} \frac{c_{i}(1-\alpha)}{\left(q[i]_{q}+\alpha\right)}(z-\zeta)^{i}+s \sum_{k=n+1}^{\infty} c\left(\sum_{j=1}^{m} d_{j} a_{k, j}\right)(z-\zeta)^{k} \text {. } \\
& \text { Since } f_{j}(z) \in M_{q} S^{*}\left(\zeta, \alpha, c_{n}\right) \text { for every } \\
& \sum_{k=n+1}^{\infty} \frac{\lambda_{k}\left(1-\sum_{i=1}^{n} c_{i}\right)(1-\alpha)}{\left(q[k]_{q}+\alpha\right)} \cdot\left(q[k]_{q}+\alpha\right) \\
& \mathrm{j}=1,2, \ldots, \mathrm{m} \text {, in view of Theorem } 10 \text {, we get } \\
& \sum_{k=n+1}^{\infty}\left(q[k]_{q}+\alpha\right) a_{k, j} \leq(1-\alpha)\left(1-\sum_{i=1}^{n} c_{i}\right), \quad j=1,2, \ldots, m . \\
& =\left(1-\sum_{i=1}^{n} c_{i}\right)(1-\alpha) \sum_{k=n+1}^{\infty} \lambda_{k} \\
& =\left(1-\sum_{i=1}^{n} c_{i}\right)(1-\alpha)(1-\lambda)
\end{aligned}
$$

Proof : We suppose that the function $f(z)$ can be expressed in the form (7.8). Then from (7.6), (7.7) and (7.9) we have

Thus we obtain

$$
f(z)=\frac{1}{z-\zeta}+\sum_{i=1}^{n} \frac{c_{i}(1-\alpha)}{\left(q[i]_{q}+\alpha\right)}(z-\zeta)^{i}+\sum_{k=n+1}^{\infty} \frac{\lambda_{k}\left(1-\sum_{i=1}^{n} c_{i}\right)(1-\alpha}{\left(q[k]_{q}+\alpha\right)}
$$

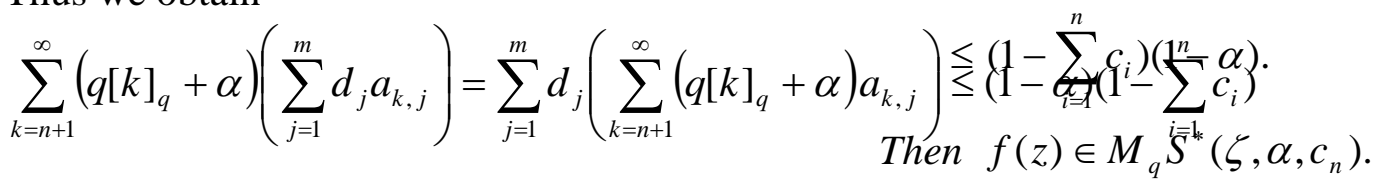

which implies that $F(z) \in M_{q} S^{*}\left(\zeta, \alpha, c_{n}\right)$.

Conversely, assuming that $f(z)$ defined by

Theorem 13: Let

$$
f_{n}(z)=\frac{1}{z-\zeta}+\sum_{i=1}^{n} \frac{c_{i}(1-\alpha)}{\left(q[i]_{q}+\alpha\right)}(z-\zeta)^{i}
$$

and

$$
f_{k}(z)=\frac{1}{z-\zeta}+\sum_{i=1}^{n} \frac{c_{i}(1-\alpha)}{\left(q[i]_{q}+\alpha\right)}(z-\zeta)^{i}+\frac{\left(1-\sum_{i=1}^{n} c_{i}\right)(1-\alpha) \lambda_{k}=\frac{\left(q[k]_{q}+\alpha\right)}{\left(q[k]_{q}+\alpha\right)}\left(z-\zeta \not 1^{k}-\sum_{i=1}^{n} c_{i}\right)(1-\alpha)}{\text { and }} a_{k} \quad(k \geq n+1),
$$$$
\text { (6.1) be in the class } M_{q} S^{*}\left(\zeta, \alpha, c_{n}\right) \text { which }
$$

\section{Setting}

$$
a_{k} \leq \frac{\left(1-\sum_{i=1}^{n} c_{i}\right)(1-\alpha)}{\left(q[k]_{q}+\alpha\right)} \quad(k \geq n+1) .
$$

for $k \geq n+1$. Then the function $f(z)$ is in the class $M_{q} S^{*}\left(\zeta, \alpha, c_{n}\right)$ if and only if it 
Corollary 4: The extreme points of the class $M_{q} S^{*}\left(\zeta, \alpha, c_{n}\right)$ are

$$
f_{n}(z)=\frac{1}{z-\zeta}+\sum_{i=1}^{n} \frac{c_{i}(1-\alpha)}{\left(q[k]_{q}+\alpha\right)}(z-\zeta)^{i}
$$

and $f_{k}(z)=\frac{1}{z-\zeta}+\sum_{i=1}^{n} \frac{c_{i}(1-\alpha)}{\left(q[i]_{q}+\alpha\right)}(z-\zeta)^{i}+\frac{(1-c)(1-\alpha)}{\left(q[k]_{q}+\alpha\right)}$

Theorem 14: Let the function $f(z)$ defined by (6.1) is in the class $M_{q} S^{*}\left(\zeta, \alpha, c_{k}\right)$. Then $f(z)$ is convex of order $\rho(0 \leq \rho \leq 1)$ in the disk $|z-\zeta|<r_{3}(\zeta, \alpha, c, \rho)$, where $r_{3}(\zeta, \alpha, c, \rho)$ is the largest value for which $\sum_{i=1}^{n} \frac{i(i+\rho)(1-\alpha) c_{i}}{\left(q[i]_{q}+\alpha\right)} r^{i+1}+\frac{k(k+\rho)(1-\alpha)\left(1-\sum_{i=1}^{n} c_{i}\right)}{\left(\left(q[k]_{q}+\alpha\right)\right.} r^{k+1} \leq(1$ ) (7.10)

for $k \geq n+1$. The result is sharp for function $f(z)$ given by (7.1).

Proof : It suffices to show that

$$
\left|\frac{(z-\zeta) f^{\prime \prime}(z)}{f^{\prime}(z)}+2\right| \leq(1-\rho)
$$

for $|z-\zeta|<r_{2}$ with the aid of Theorem 2 .

Then by using the same technique in the proof of Theorem 8 we can complete the proof.

\section{Reference}

[1] G. A. Anastassiou and S. G. Gal, Geometric and approximation properties of some singular integrals in the unit disk, Journal of Inequalities and Applications, Article ID 17231, 19 pages, 2006.
[2] G. A. Anastassiou and S. G. Gal, Geometric and approximation properties of generalized singular integrals in the unit disk, J. Korean Math. Soc, 43(2), 425-443 (2006).

[3] A. Aral, On the generalized Picard and Gauss Weierstrass singular integrals, Journal of Computational Analysis and - Appplications, ${ }^{k}$ \&(3), 249--261 (2006).

[4] A. Aral, V. Gupta and R. P. Agarwal, Applications of $q$-Calculus in Operator Theorem, Springer, New York, NY, USA, 2013.

[5] A. Aral and V. Gupta, On q Baskakov type operators, Demonstratio Mathematica, 42(1),109--122 (2009).

[6] A. Aral and V. Gupta, Generalized $q$ -Baskakov operators, Mathematica Slovaca, 61(4), 619--634 (2011).

[7] A. Aral and V. Gupta, On the (Duprmeyer type modification of the Baskakov type operators, Nonlinear Analysis: Theory, Methods \& Applications, 72( 3-4), 1171--1180, 2010.

[8] H. Exton, $q$-Hypergeometric Functions and Applications, Ellis Horwood Series: Mathematics and Its Applications, Ellis Horwood, Chichester, UK, 1983.

[9] F. H. Jackson, On $q$ - functions and a certain difference operator, Transactions of the Royal Society of Edinburgh, 46(1908), 253-281.

[10] F. H. Jackson, On $q$-definite integrals, The Quarterly Journal of Pure and Applied Mathematics, 41(1910), 193-203.

[11] A. Mohammed and M. Darus, Ageneralized operator involving the qhypergeometric function, Matematiki Vesnik 65(4), 454--465 (20 13). 